\title{
A Fully Self-Powered Vibration Monitoring System Driven by Dual-Mode Triboelectric Nanogenerators
}

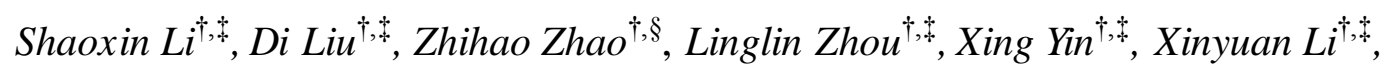
Yikui Gao ${ }^{\dagger, \#}$, Chuguo Zhang ${ }^{\dagger, \ddagger}$, Qing Zhang ${ }^{\dagger}$, Jie Wang ${ }^{\dagger, \star, \#}$, Zhong Lin Wang ${ }^{\dagger, \downarrow, \perp}$

${ }^{\dagger}$ Beijing Institute of Nanoenergy and Nanosystems, Chinese Academy of Sciences, Beijing 100083, P. R. China

†College of Nanoscience and Technology, University of Chinese Academy of Sciences, Beijing 100049, P. R. China

${ }^{\S}$ School of Materials, Sun Yat-sen University, Guangzhou 510275, P. R. China

${ }^{\#}$ Center on Nanoenergy Research, School of Physical Science and Technology,

Guangxi University, Nanning 530004, P. R. China

${ }^{\perp}$ School of Materials Science and Engineering, Georgia Institute of Technology, Atlanta, GA 30332, USA

\section{Corresponding Authors}

*Email: wangiie@binn.cas.cn

*Email: zhong.wang@ mse.gatech.edu

\section{Author Contributions}

Shaoxin Li, Di Liu, Zhihao Zhao contributed equally to this work. 
Supporting Note 1

When the vibration amplitude is fixed and the vibration speed changes, the peak current has a positive correlation with velocity, which can be explained by the following formula:

$I_{\mathrm{AC}}=\frac{d Q_{\mathrm{AC}}}{d t}=\frac{d Q_{\mathrm{AC}}}{d x} \cdot \frac{d x}{d t}=\frac{d Q_{\mathrm{AC}}}{d x} \cdot v$

where $I_{\mathrm{AC}}$ is the short-circuit current of AC-TENG, $d Q_{\mathrm{AC}}$ the amount of transferred charge of each vibration within safe region, $d x$ the vibration amplitude of slider $(0$ $\mathrm{mm} \leq d x \leq 10 \mathrm{~mm}), \quad v$ the vibration velocity of slider. 


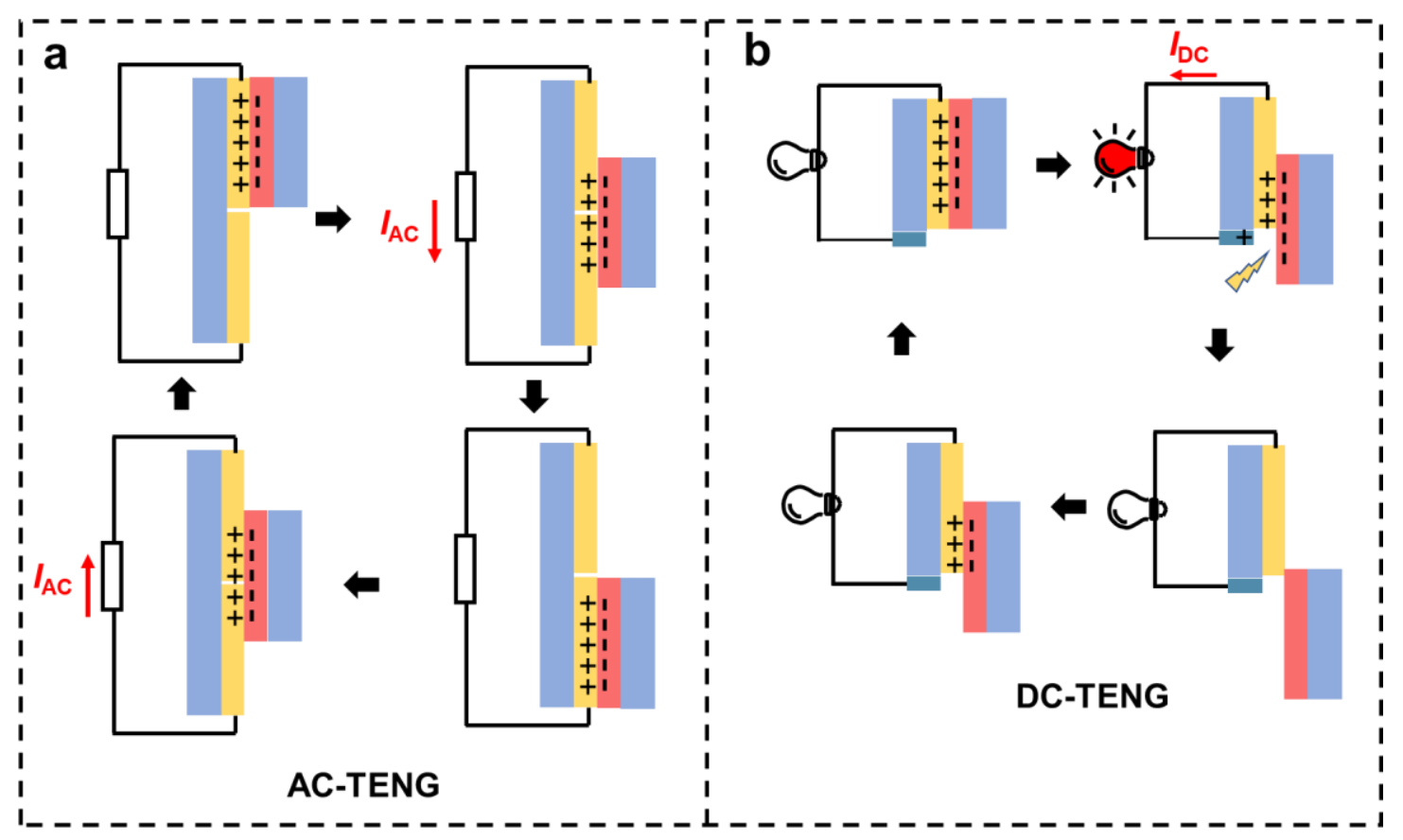

Figure S1. The working mechanism of (a) AC-TENG and (b) DC-TENG.

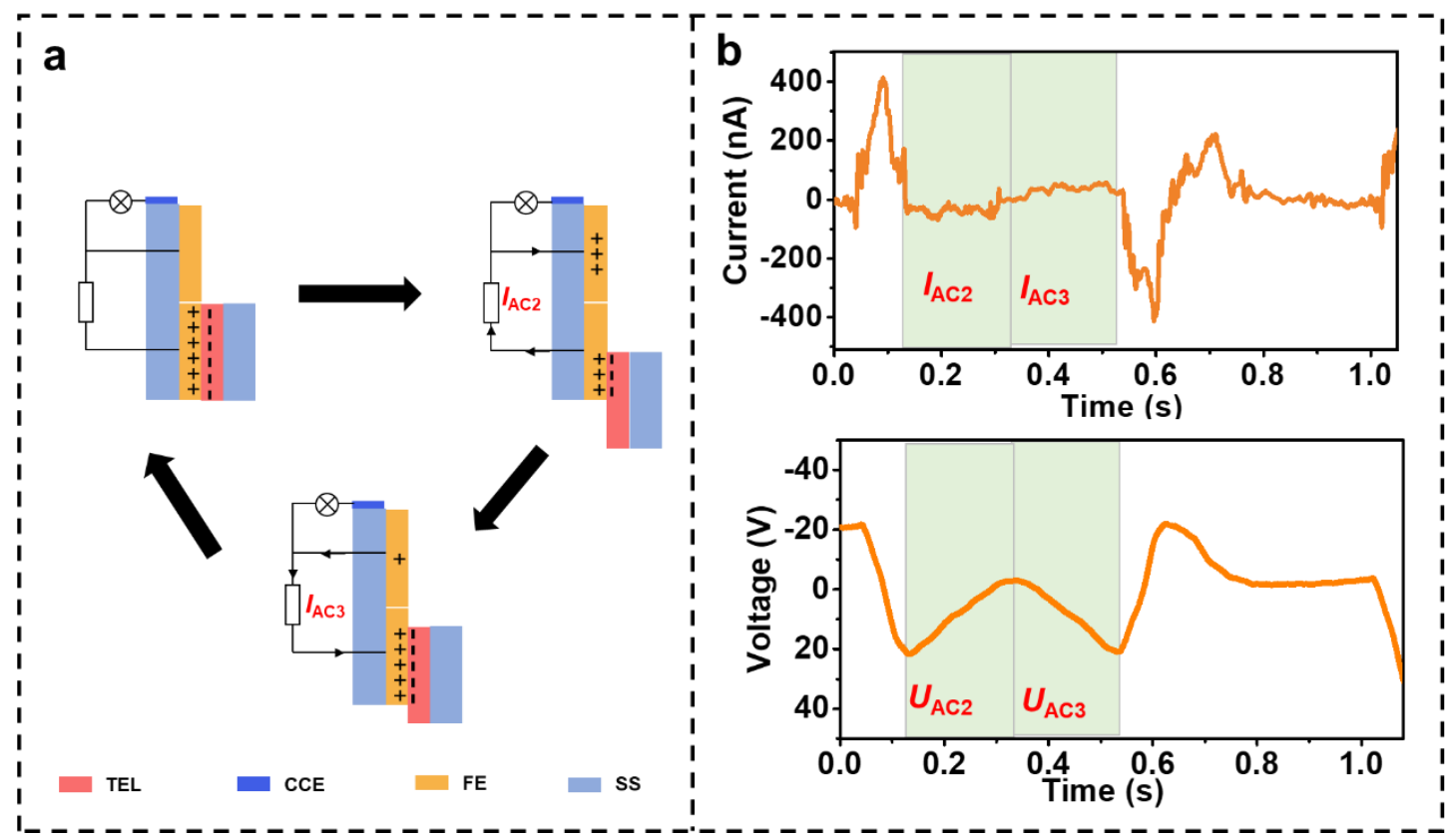

Figure S2. The output performance when the slider exceeds the amplitude threshold but without CCE. (a) The slider moves out of the FE but without CCE. (b) The electrical signals when the slider exceeds the amplitude threshold but without CCE. 


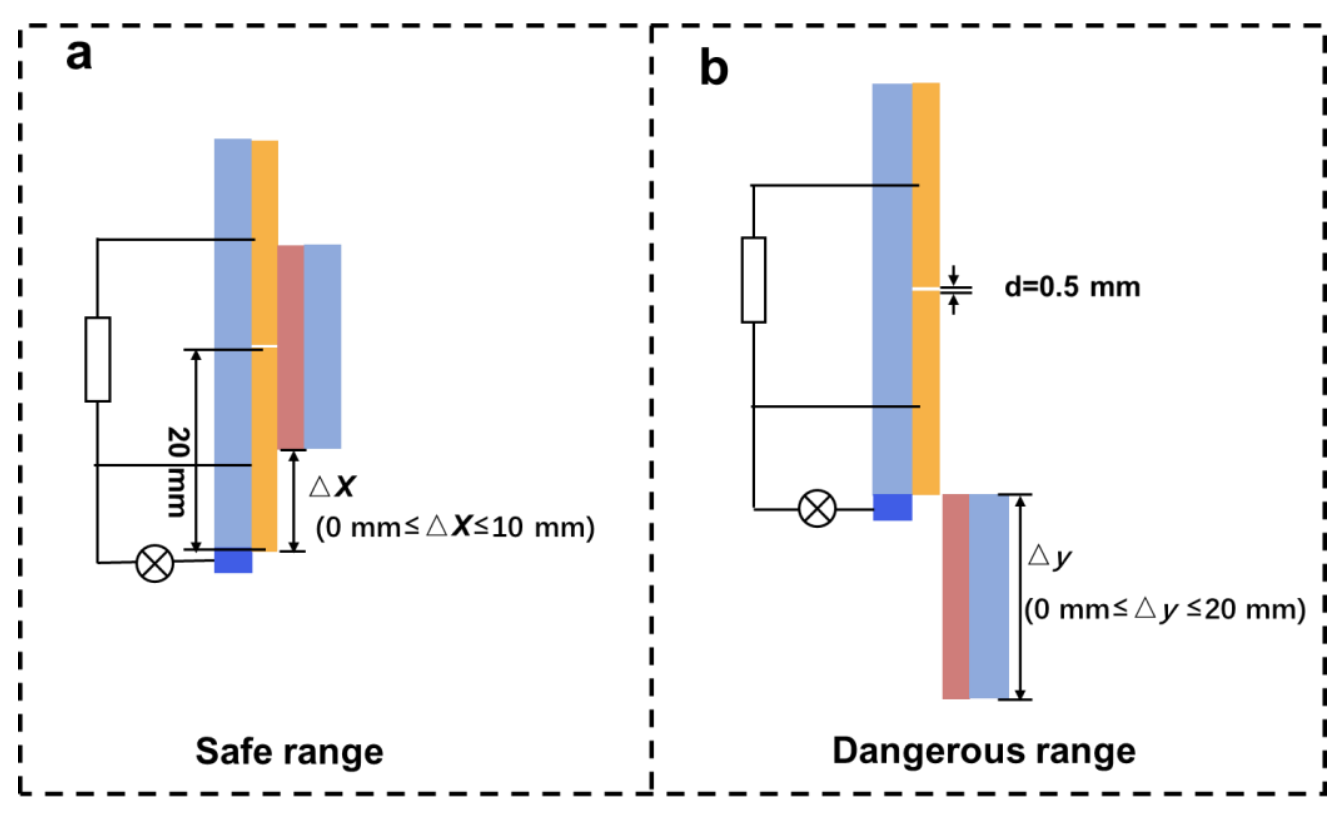

Figure S3. Two motion ranges of AC/DC-TENG.

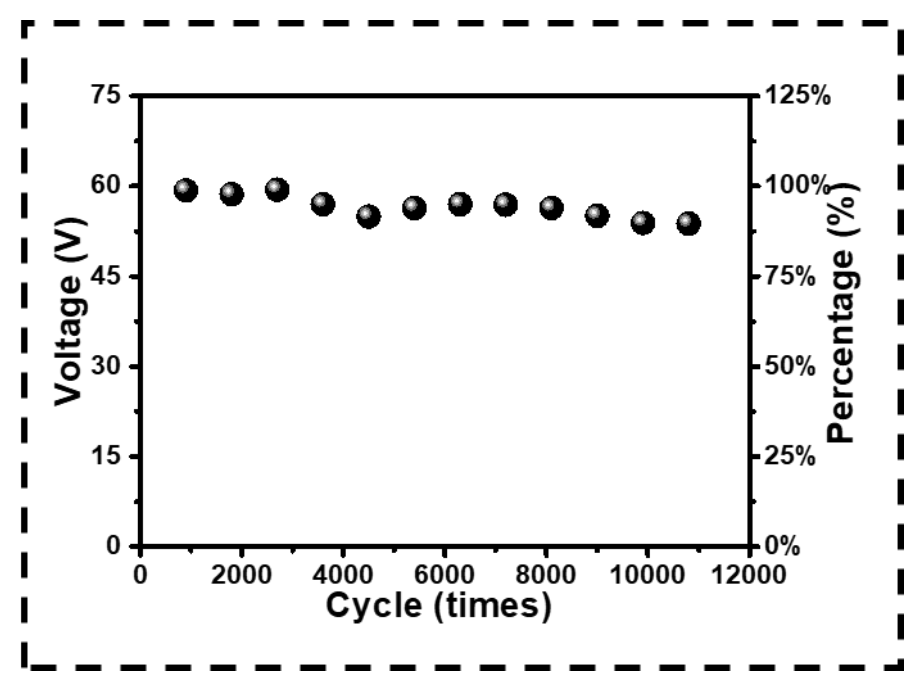

Figure S4. The stability of AC/DC-TENG. 


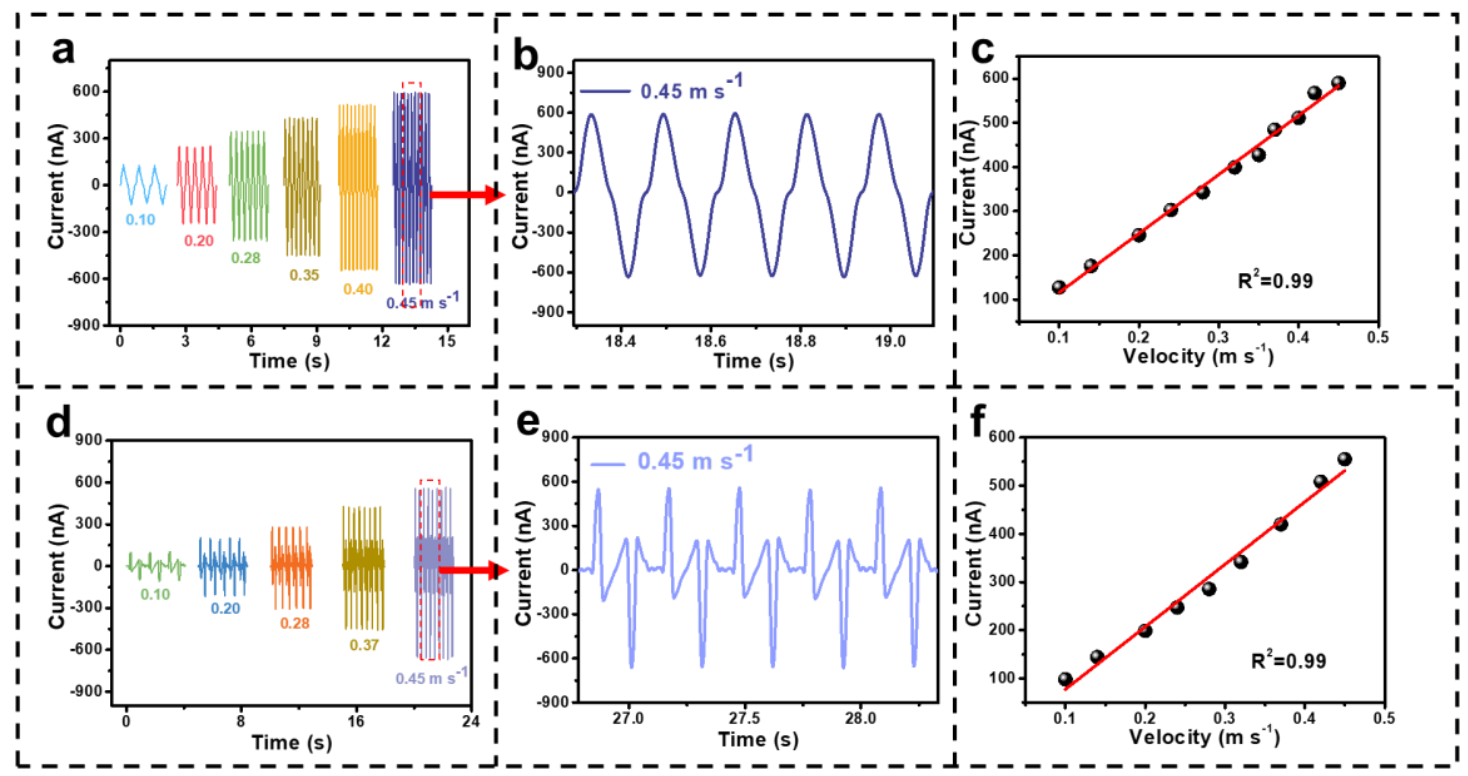

Figure S5. The relationship between output current and velocity of AC/DC-TENG. (a)

The $I_{\mathrm{AC}}$ of AC/DC-TENG at the vibration amplitude of $8 \mathrm{~mm}$. (b) The enlarged image of $I_{\mathrm{AC}}$ of the AC/DC-TENG at the velocity of $0.45 \mathrm{~m} \mathrm{~s}^{-1}$. (c) Dependence of the $I_{\mathrm{AC}}$ on the corresponding velocity at the vibration amplitude of $8 \mathrm{~mm}$. (d) The complete current signals of AC/DC-TENG at the vibration amplitude of $29.5 \mathrm{~mm}$. (e) The enlarged image of output current of the AC/DC-TENG at the velocity of $0.45 \mathrm{~m} \mathrm{~s}^{-1}$. (f) Dependence of the current on the corresponding velocity at the vibration amplitude of $29.5 \mathrm{~mm}$.

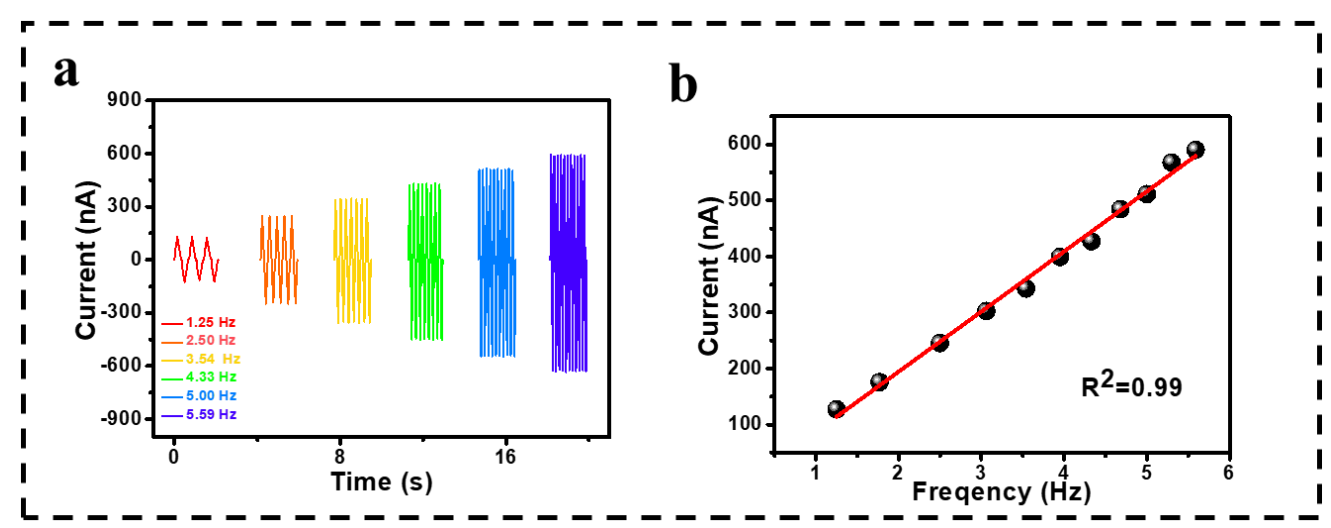

Figure S6. The relationship of vibration frequency and the output current. (a) Output 
alternating current of AC/DC-TENG at several vibration frequency in the case of 8 mm. (b) Dependence of the current on the corresponding frequency in the case of 8 mm.

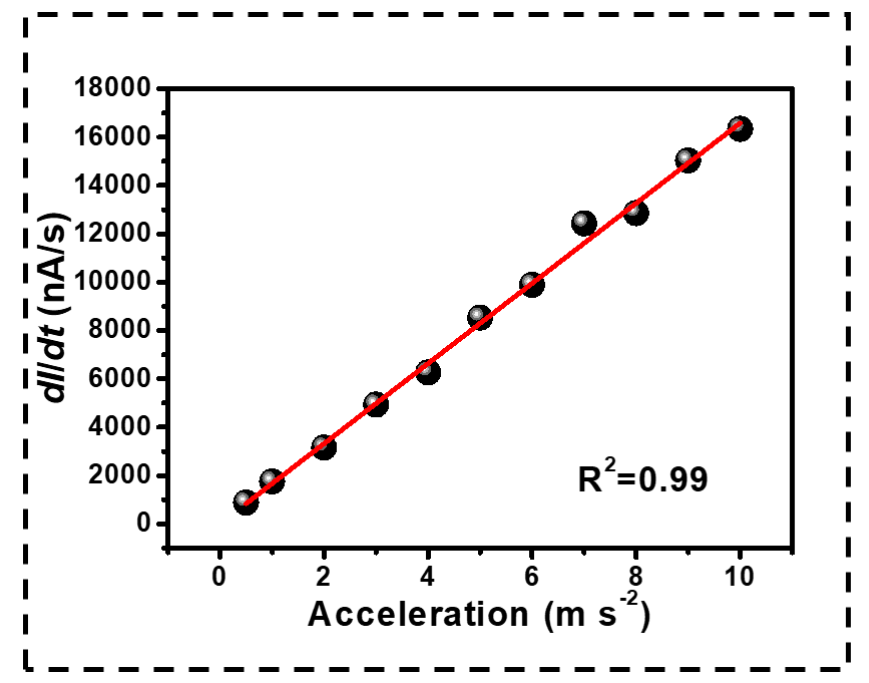

Figure S7. The linear relationship between $d I / d t$ and vibration acceleration.

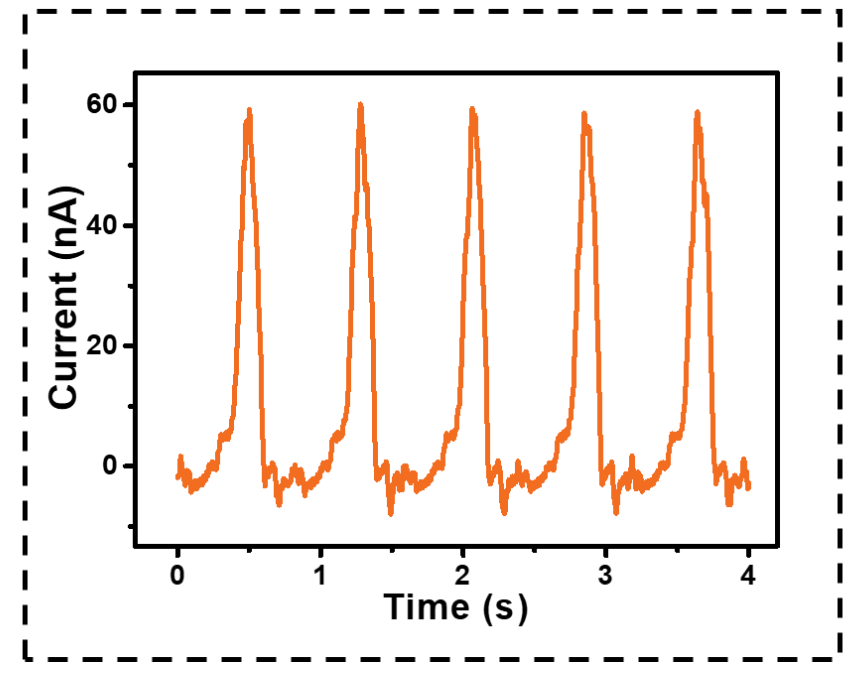

Figure S8. The DC signal of DC-TENG. 


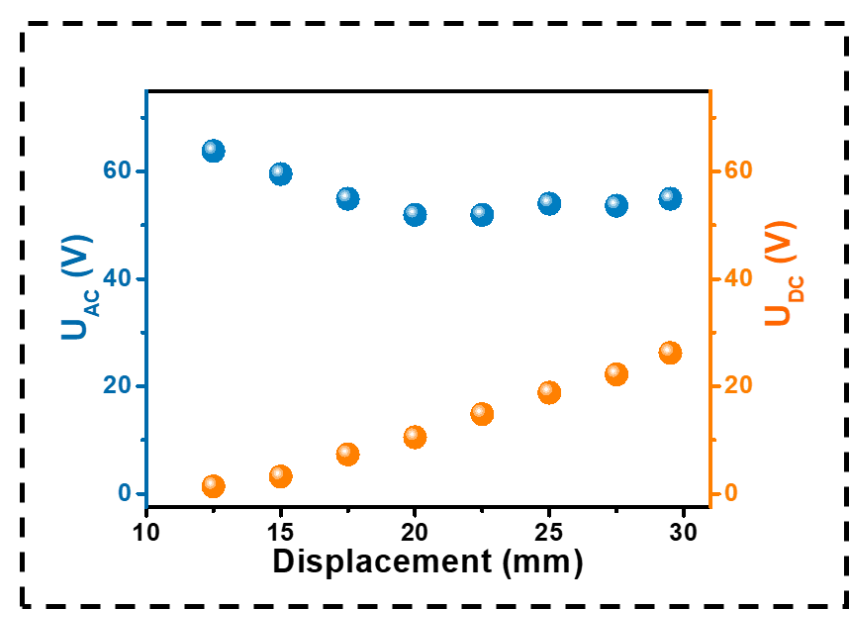

Figure S9. The AC and DC output of AC/DC-TENG when the vibration amplitude over the threshold.

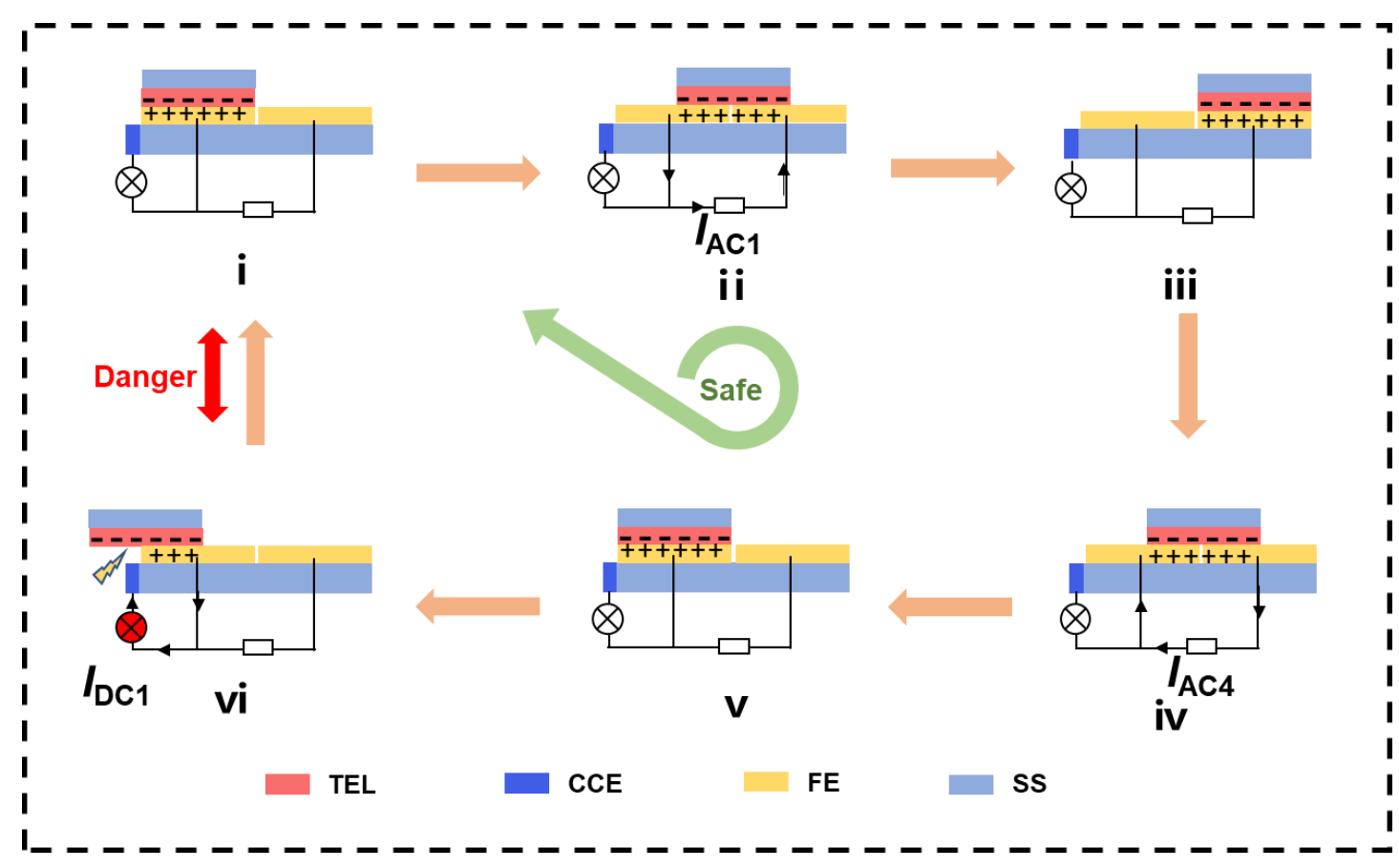

Figure S10. Working mechanism of the AC/DC-TENG oscillates in the horizontal direction. 


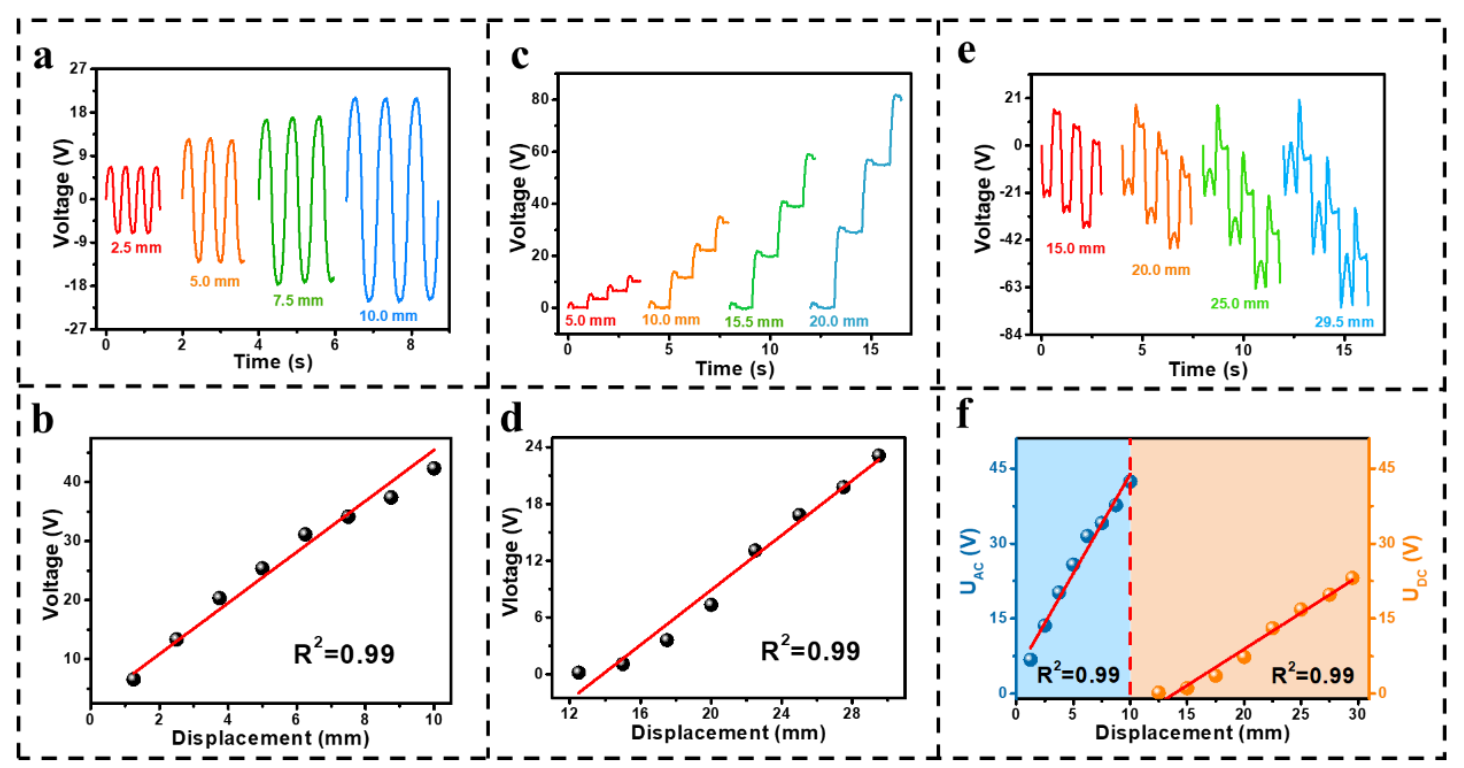

Figure S11. The AC/DC-TENG oscillates in the horizontal direction. (a) The $V_{\mathrm{AC}}$ of AC/DC-TENG within the safety zone. (b) The relationship between the $V_{\mathrm{AC}}$ and the vibration amplitude in the case of safety range. (c) The $V_{\mathrm{DC}}$ of AC/DC-TENG when the vibrate amplitude over the threshold. (d) The relationship between the $V_{\mathrm{DC}}$ and the corresponding amplitude in the case of dangerous zone. (e) The complete voltage signals of AC/DC-TENG when the vibration amplitude over the threshold. (f) That linear relationship between $V_{\mathrm{AC}}$ and $\Delta x$ or $V_{\mathrm{DC}}$ and $\Delta y$ in safe zone or dangerous zone, respectively.

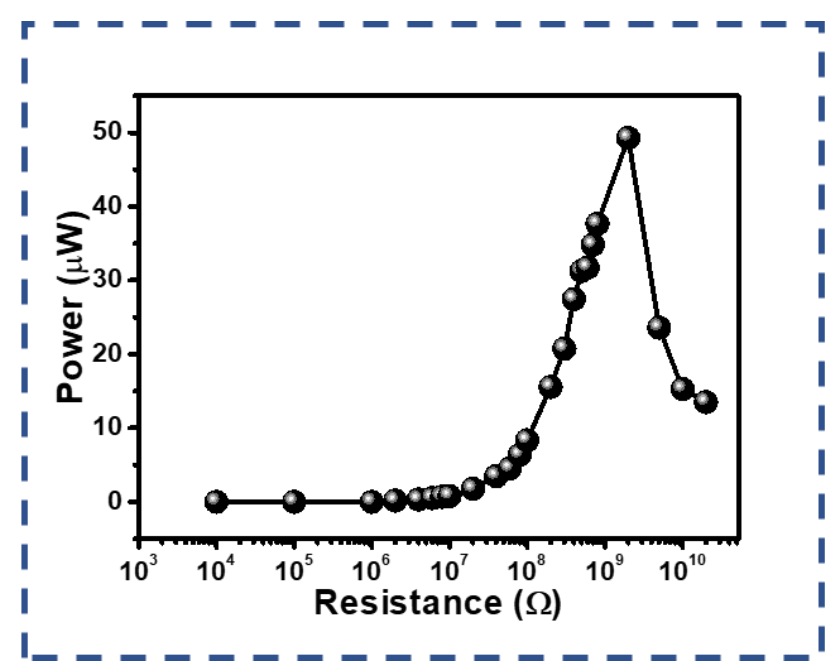


Figure S12. The output power performance of AC-TENG. 